\title{
Application of Vibration and Oil Analysis for Reliability Information on Helicopter Main Rotor Gearbox*
}

\author{
Muhamad MURRAD** and M. Salman LEONG*** \\ ${ }^{* *}$ Business \& Advanced Technology Centre, Universiti Teknologi Malaysia, \\ Jalan Semarak, 54100 Kuala Lumpur, Malaysia \\ E-mail: muhamad_murrad@hotmail.com \\ *** Institute of Noise \& Vibration, Universiti Teknologi Malaysia, \\ Jalan Semarak, 54100 Kuala Lumpur, Malaysia \\ E-mail: salman@citycampus.utm.my
}

\begin{abstract}
Based on the experiences of the Malaysian Armed Forces (MAF), failure of the main rotor gearbox (MRGB) was one of the major contributing factors to helicopter breakdowns. Even though vibration and oil analysis are the effective techniques for monitoring the health of helicopter components, these two techniques were rarely combined to form an effective assessment tool in MAF. Results of the oil analysis were often used only for oil changing schedule while assessments of MRGB condition were mainly based on overall vibration readings. A study group was formed and given a mandate to improve the maintenance strategy of S61-A4 helicopter fleet in the MAF. The improvement consisted of a structured approach to the reassessment/redefinition suitable maintenance actions that should be taken for the MRGB. Basic and enhanced tools for condition monitoring (CM) are investigated to address the predominant failures of the MRGB. Quantitative accelerated life testing (QALT) was considered in this work with an intent to obtain the required reliability information in a shorter time with tests under normal stress conditions. These tests when performed correctly can provide valuable information about MRGB performance under normal operating conditions which enable maintenance personnel to make decision more quickly, accurately and economically. The time-to-failure and probability of failure information of the MRGB were generated by applying QALT analysis principles. This study is anticipated to make a dramatic change in its approach to $\mathrm{CM}$, bringing significant savings and various benefits to MAF.
\end{abstract}

Key words: Reliability Information, Condition Monitoring, Probability of Failure, Helicopter Main Rotor Gearbox, Quantitative Accelerated Life Testing, Oil and Vibration Analysis

\section{Introduction}

Condition monitoring (CM) systems and condition based maintenance widespread are increasingly being used in the rotorcraft industry. This is due to the need of providing safe operation of crucial helicopter components such as rotor, engine, gearbox and drive train as well as their cost-efficient maintenance policies, which are mandatory in today's scenario, including Malaysian Armed Forces (MAF). Since the beginning of 1990s, MAF has tried to strongly reduce military expenses. Demands are for helicopter emphasizing high reliability for minimal unscheduled maintenance, with reduced life-cycle costs, durability for longer 
interval between overhaul and maintainability for simple and fast maintenance operations. These demands can be met if the primary goals of a comprehensive CM program are fulfilled.

This paper reports on initiative to measure reliability with application of quantitative accelerated life testing (QALT) principles to the condition monitored data ${ }^{(1)}$. The expected life of the main rotor gearbox (MRGB) can be estimated by computing time-to-failure data under accelerated stress condition without undergoing failure condition. This is important as in MRGB situation, such time-to-failure data, although not difficult, is very expensive to obtain. Mathematical models can then be used to extrapolate a use-level cumulative distribution function to determine probability of failure and other reliable information about the expected life of the MRGB with confidence bounds. Commercially available software was used to perform the analysis on the data collected in support of this study ${ }^{(2)}$.

This paper provides an overall view to show such a tool can potentially provide significant enhancement in determining reliability of MRGB in today's CM programs. With collection of adequate and meaningful data on MRGB performance in the field, its reliability can be measured, tracked and hopefully improved. The expected result is to decrease the system complexity by providing the end user with a simple tool to predict failure based on the health in the system. Moreover, addition of enhanced measurement technology, oil analysis, will expand the defect coverage. The objectives of this paper are:

- To review the strengths of the enhanced oil analysis to the CM of the MRGB (CM is primarily a non-preventive maintenance process).

- To describe the strength of the combined proactive and predictive maintenance strategies for the MRGB in pursuit for a CM program.

- To anticipate probability of failure of MRGB by QALT analysis based on computed time-to-failure information analyzed from the enhanced oil and vibration data.

\section{Existing In-House Maintenance Practice}

The development of highly reliable health-monitoring system is one technology recommended for reducing the catastrophic failure of crucial helicopter components such as MRGB. The system is much needed in the MAF as the helicopter maintenance record for the past 18 months showed the MRGB of S-61A Sikorsky (known as NURI in MAF) helicopters appeared to be the biggest contributing factor to NURI unexpected failures or breakdowns. These unexpected problems affect operation and maintenance costs, pilot training flights hours, repair and overhaul time, spare parts inventory and aircraft uptime. The current maintenance operations involve a mixture of time based and condition based maintenance. The present predictive and proactive capabilities are very little, limiting to the current techniques of spectrometric oil analysis program (SOAP) and vibration monitoring. Even though the data are collected in preventive maintenance activities performed at fixed flight maintenance intervals as stated in the original equipment manufacturer's maintenance instructions, this data is not stored for further analysis. The maintenance department has relied on individual SOAP and vibration monitoring to make maintenance decisions on MRGB. Both tools are rarely combined to form an effective union. Vibration analysis activities typically reside in the vibration monitoring group, while SOAP usually resides with the lubrication monitoring group. The SOAP is used to schedule oil changes rather than interpreting wear conditions. Condition assessments of the MRGB are primarily based on the overall vibration readings of the MRGB.

As practiced elsewhere, the maintenance department takes actions to retain MRGB in serviceable condition or to restore it to serviceability. This enables the helicopters to be available. Realising MRGB and its components will degrade and sometimes fail in service, the maintenance department has placed its role to be able to anticipate and control this degradation, to avoid failures if possible and, if a failure does occurs, to get the equipment 
back up and running in as short a time as possible. Unfortunately, the high failures suggested that these intentions had not been fully realised.

\section{Review of the Condition Monitoring Techniques Used}

Oil analysis has recognised advantages, as it can provide direct and early information on wear modes and the component's condition. In fact, in many instances it has been proven to be a leading indicator of active component wear, compared to vibration analysis. In addition, oil analysis has certain advantages in monitoring low-speed machinery (less than 5 $\mathrm{rpm}$ ), where it is usually difficult to apply vibration analysis techniques. However, oil analysis cannot effectively uncover all manners of failure mechanisms on its own. For this reason, a comprehensive CM program is best for the reliability of the MRGB. Noria Corporation's Oil Analysis training ${ }^{(3)}$ has suggested that for early abnormal conditions detection and for managing important failure root causes, both oil analysis and vibration analysis are necessary and vital parts to a comprehensive CM program for the MRGB.

\subsection{Enhanced Oil Analysis}

Oil analysis is a broad field comprised of hundreds of individual tests that provide meaningful benefit by assessing one or more properties of a lubricant or equipment. For the MRGB, there are tests that provide information about new oils where the fluid's chemical, physical or lubricating properties are evaluated. Used oil analysis test, the focus of this paper, differs substantially from new oil test. The objective of used oil analysis test is to evaluate the condition of oils that are in service and to evaluate the condition of the MRGB being lubricated. The lubricant serves merely as the carrier of the information that is generated by the MRGB in the form of contamination or wear debris.

Essentially, oil analysis is performed to improve the quality of the MRGB and lubrication maintenance decisions. Three categories of the oil analysis performed are:

- Fluid Properties Analysis. Deals with the assessment of the chemical, physical and additive properties of the MRGB oil.

- Contaminant Analysis. Foreign matters that enter the MRGB system from the environment, or are generated internally, are contaminants. Contamination compromises the MRGB reliability and promotes lubricant failure. Oil analysis ensures that goal-driven targets for contamination control are maintained.

- Wear Debris Analysis. When MRGB components wear, they generate debris. Monitoring and analyzing the generated debris enables analyst to detect and evaluate abnormal conditions for effective maintenance decisions to be made and implemented.

Enhanced oil tests and benefits to the categories of oil analysis for the MRGB are shown:

Table 1. Enhanced Oil Tests and Benefits

\begin{tabular}{|c|c|c|c|}
\hline \multirow{2}{*}{$\begin{array}{l}\text { What is being } \\
\text { analyzed }\end{array}$} & 1. Fluid Properties & 2. Contamination & 3. Wear Debris \\
\hline & $\begin{array}{l}\text { Physical \& chemical } \\
\text { properties of new/used oil }\end{array}$ & $\begin{array}{l}\text { Fluid \& MRGB destructive } \\
\text { contaminants }\end{array}$ & $\begin{array}{l}\text { Presence/identification of } \\
\text { wear particles }\end{array}$ \\
\hline \multicolumn{4}{|l|}{ Particle Count } \\
\hline \multicolumn{4}{|l|}{ Moisture } \\
\hline \multicolumn{4}{|l|}{ Viscosity } \\
\hline \multicolumn{4}{|l|}{ Acid Number } \\
\hline \multicolumn{4}{|l|}{ Elemental } \\
\hline \multirow[t]{2}{*}{ Strategy } & PROACTIVE & PROACTIVE & PREDICTIVE \\
\hline & Primary benefit & ( Minor benefit & No benefit \\
\hline
\end{tabular}

Reprinted from: Drew Troyer and Jim Fitch, Oil Analysis Basics, 2001, p.62 ${ }^{(4)}$ 


\subsection{Vibration Analysis}

Vibration analysis is an established predictive maintenance procedure and as a support for MRGB maintenance decisions. The overall vibration signal emanating from the MRGB is a prime candidate for monitoring, as many of the failures that occur due to the rotating components (gears, bearings) show their symptoms as changes in the frequencies and amplitudes of vibration signatures ${ }^{(5)}$. As a general rule, MRGB does not break down or fail without some form of warning, which is indicated by an increased vibration level. By measuring and analyzing the vibration of MRGB, it is possible to determine both the nature and severity of the defect, and hence predict the MRGB failure.

\section{Review of QALT Analysis}

This paper presents an overview of basic concepts in QALT analysis used for reliability information on the MRGB. In traditional life data analysis, manufacturers analyze life data of a product's sample operating under normal operating conditions in order to quantify the life characteristics of the product and make predictions about all of the products in the population. Over the years, if they wish to obtain reliability results for their products more quickly, they may use accelerated life testing to force their products to fail more quickly than they would under normal use conditions. By accelerating the failures under accelerated stress conditions, manufacturers may understand better the failure modes and life characteristics of the products.

An understanding of the principles behind QALT analysis is needed before performing an analysis on the collected oil and vibration data. If viewed from a traditional life data analysis perspective, this study is not a QALT. However, its analysis will require this study to employ the fundamental principles of QALT analysis. The measured value of each oil and vibration characteristic as measured at interval flight hours can be viewed as the random variable (the time value in standard QALT analysis) affected by the ageing process (the stress value in standard QALT analysis). In other words, the stress on each measurement taken is the flight hours and the random variable (what is traditionally termed as time-to-failure) is the value of the measured characteristic of each oil and vibration.

In brief, the theoretical description of the QALT related to this case study is as follows:

\subsection{Select a Life Distribution}

The first step in performing QALT is to choose an appropriate life distribution. The Lognormal distribution, which requires more involved calculations, is more appropriate for most uses. The Lognormal distribution is given by:

$$
f\left(T^{\prime}\right)=\frac{1}{\sigma_{T^{\prime}} \sqrt{2 \pi}} e^{-\frac{1}{2}\left(\frac{T^{\prime}-\bar{T}^{\prime}}{\sigma_{T^{\prime}}}\right)^{2}}
$$

where:

$T^{\prime}=\ln T$, and where the $T$ s are the times-to-failure

$\bar{T}^{\prime}=$ mean of the natural logarithms of the times to failure

$\sigma_{T^{\prime}}=$ standard deviation of the natural logarithms of the times to failure

\subsection{Select a Life-Stress Relationship}

After selected an underlying life distribution appropriate to the oil and vibration data, the second step is to select (or create) a model that describes a characteristic point or a life characteristic of the distribution from one stress level to another. The General Log-Linear (GLL) model describes a life characteristic as a function of a vector of $n$ stresses, or $X=\left(X_{1}\right.$, $X_{2} \ldots X_{\mathrm{n}}$ ). Mathematically the model is given by, 


$$
L(\underline{X})=e^{\alpha_{0}+\sum_{j=1}^{n} \alpha_{j} X_{j}}
$$

where:

$\alpha_{j}$ are model parameters

$X$ is a vector of $n$ stresses

\subsection{GLL-Lognormal}

The GLL-Lognormal model can then be derived as follows:

$$
f(t, \underline{X})=\frac{1}{t \sigma_{T^{\prime}} \sqrt{2 \pi}} e^{-\frac{1}{2}\left(\frac{T^{\prime}-\alpha_{0}-\sum_{j=1}^{n} \alpha_{j} X_{j}}{\sigma_{T^{\prime}}}\right)^{2}}
$$

\subsection{Descriptions about Quantitative Estimated Validity}

QALT analysis uses life data obtained under accelerated conditions to extrapolate an estimated probability density function ( $p d f$ ) for the MRGB under normal use conditions. It employs overstress acceleration to speed up the time-to-failure for the MRGB under test. The flight hours, the only factor that causes the MRGB to fail under normal conditions is increased in order to stimulate the MRGB to fail more quickly. Data from this type of test require special accelerated life data analysis techniques, which include a mathematical model to "translate" the overstress $p d f s$ to normal use conditions.

4.4.1. Stress Type and Stress Level For QALT in this paper, the increased flight hour is one stress type that causes the MRGB to fail under normal use conditions. This stress is applied at various increased levels and measures the times-to-failure for the MRGB under accelerated test conditions, in order to stimulate the MRGB under test to fail more quickly. In this case study, the stress type is flight hours and the accelerated stress levels are $50 \mathrm{~K}$ (knots), $75 \mathrm{~K}$, $100 \mathrm{~K}$ and $125 \mathrm{~K}$. The use stress level is $25 \mathrm{~K}$. Using the life data obtained at each accelerated stress level, standard life data analysis techniques can be used to estimate the parameters for the life distribution (lognormal) that best fits the data at each stress level. This results in an overstress $p d f$ for each accelerated stress level. Another mathematical model, the life-stress relationship, is then required to estimate the $p d f$ at the normal use stress level

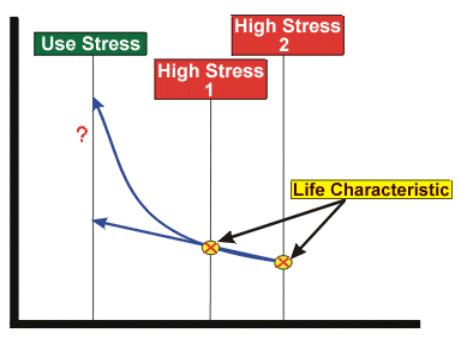

Fig 1. Life Characteristics based on the characteristics of the $p d f s$ at each accelerated stress level.

4.4.2. Life-Stress Relationship A life-stress relationship that fits the type of data being analyzed, GLL model, is chosen to extrapolate a use level $p d f$ from life data obtained at increased stress levels. This model describes the path of a life characteristic of the distribution from one stress level to another as shown in Fig 1. The life characteristic is of a life measure, the median, expressed as a function of stress.

4.4.3. Probability of Failure Given Time Once the parameters to fit a life distribution and a life-stress relationship to a particular data set have been calculated, the probability that MRGB will be failed at a particular point in time under normal use conditions. Probability of failure is also known as "unreliability" and it is the reciprocal of the reliability. For example, there is a $99 \%$ chance that the MRGB will be failed after 2000 flight hours of operation at a given stress level (and a 1\% chance that it will operate successfully). 


\section{Data Collection and Computation}

The laboratory results of the enhanced oil tests on the MRGB are as follows:

Table 2. The MRGB Enhanced Oil Data

\begin{tabular}{|c|c|c|c|c|c|c|c|c|c|}
\hline \multirow{3}{*}{ Oil Tests } & \multicolumn{4}{|c|}{ Limits / Targets } & \multirow{2}{*}{\multicolumn{5}{|c|}{$\begin{array}{l}\text { Samples taken at following } \\
\text { flight hours }(\mathrm{H}) \text { intervals }\end{array}$}} \\
\hline & \multicolumn{2}{|c|}{ Lower } & \multicolumn{2}{|c|}{ Upper } & & & & & \\
\hline & Critical & Caution & Caution & Critical & $25 \mathrm{H}$ & $50 \mathrm{H}$ & $75 \mathrm{H}$ & $100 \mathrm{H}$ & $125 \mathrm{H}$ \\
\hline Vis $40^{\circ} \mathrm{C}$, cSt & 26 & 29 & 36 & 39 & 31 & 32 & 33 & 32 & 32 \\
\hline Acid Number, mg/g & $*$ & * & 0.6 & 1.1 & 0.1 & 0.2 & 0.2 & 0.3 & 0.2 \\
\hline Moisture, ppm & $*$ & $*$ & 400 & 800 & 700 & 650 & 600 & 510 & 425 \\
\hline \multicolumn{10}{|l|}{ Wear Metals, $\boldsymbol{\mu} \mathrm{m}$} \\
\hline $\mathrm{Fe}$ & $*$ & * & 0.96 & 1.12 & 0.8 & 0.9 & 0.8 & 1.1 & 3.0 \\
\hline $\mathrm{Sn}$ & $*$ & * & 0.74 & 0.78 & 0.6 & 0.5 & 0.7 & 0.8 & 1.4 \\
\hline $\mathrm{Cu}$ & $*$ & $*$ & 0.56 & 0.62 & 0.4 & 0.3 & 0.5 & 0.6 & 1.6 \\
\hline \multicolumn{10}{|l|}{ Contamination, ppm } \\
\hline $\mathrm{Si}$ & $*$ & $*$ & 23 & 34 & 8 & 11 & 9 & 12 & 8 \\
\hline $\mathrm{Al}$ & $*$ & $*$ & 70 & 100 & 32 & 40 & 38 & 77 & 109 \\
\hline Particle Count (ISO) & $*$ & $*$ & $15 / 11$ & $17 / 14$ & $15 / 11$ & $14 / 12$ & $15 / 12$ & $18 / 14$ & $24 / 21$ \\
\hline
\end{tabular}

Note: * - No lower limits, $\mu \mathrm{m}$ - microns

The vibration readings obtained are as follows:

Table 3. The MRGB Vibration Data (Max $0.2 \mathrm{ips}$ )

\begin{tabular}{|c|c|c|c|c|}
\hline \multirow{2}{*}{ Flight Hrs } & \multicolumn{4}{|c|}{ ips } \\
\cline { 2 - 5 } & Ground & Hover & $80 \mathrm{~K}$ & $110 \mathrm{~K}$ \\
\hline 25 & 0.19 & 0.19 & 0.22 & 0.22 \\
\hline 50 & 0.20 & 0.19 & 0.25 & 0.22 \\
\hline 75 & 0.18 & 0.12 & 0.08 & 0.06 \\
\hline 100 & 1.59 & 0.12 & 0.08 & 0.06 \\
\hline 125 & 0.28 & 0.10 & 0.11 & 0.10 \\
\hline
\end{tabular}

Note: ips - inch per sec, K - knots

Considering the overhaul lifetime for MRGB is of 2000 flight hours. The QALT was performed based on data in Table 2 and Table 3. The probability that the MRGB will be out of compliance or fails, at or after the time period, was determined using the Lognormal distribution along with a General Log-Linear model. Plotting the Life (Oil/Vibration Data) $v s$. Stress (Flight Hours) with $90 \%$ two-sided confidence intervals resulted in the probabilities of failure for each test as shown in Appendix. For example, as can be seen from the plot in Figure 1 of Viscosity $40^{\circ} \mathrm{C} v$ s. Flight Hours, a slight increase in the characteristic is noted. The probability of failure based on viscosity at or after until 2000 flight hours is $0 \%$. This is only a probability and subsequent data after 125 flight hours may affect the percentage. No maintenance action may be required based on viscosity.

Taking another example from the plot in Figure 5 of wear metal, Sn vs. Flight Hours, there is a noticeable increase in the characteristic. The probability of the wear metal, Sn, being above the limit at or after 2000 flight hours is $99.99 \%$. Having identified Sn as one of the main causes of failure, appropriate corrective actions may be required.

Once the parameters of the underlying life distribution and stress-life relationship have been estimated, the required reliability information for the MRGB can be derived as shown in Table 4: 
Table 4. Reliability Information (Probability of Failure at or after 2000 Flight Hours)

\begin{tabular}{|l|c|c|c|c|c|c|c|c|c|c|}
\hline \multirow{2}{*}{$\begin{array}{c}\text { Refer Figures } \\
\text { in Appendix }\end{array}$} & Vis & AN & Moist & Fe & Sn & Cu & Si & Al & PC & Vibe \\
\cline { 2 - 11 } & Fig 2 & Fig 3 & Fig 4 & Fig 5 & Fig 6 & Fig 7 & Fig 8 & Fig 9 & Fig 10 & Fig 11 \\
\hline Below Limit (\%) & 0 & $*$ & $*$ & $*$ & $*$ & $*$ & $*$ & $*$ & $*$ & $*$ \\
\hline Above Limit (\%) & 0 & 0 & 0 & 99.52 & 99.99 & 99.98 & 0 & 100 & 99.15 & 98.67 \\
\hline $\begin{array}{l}\text { Appropriate } \\
\text { Corrective Action } \\
\text { Required }\end{array}$ & No & No & No & Yes & Yes & Yes & No & Yes & Yes & Yes \\
\hline
\end{tabular}

Note: * - No lower limits, PC - Particle count

There are many advantages using the QALT figures or plots in the Appendix. Beside probability of failure, the same plots may display some additional results such as:

- Reliability Given Time: The probability that the MRGB will operate successfully at a particular point in time under normal use conditions.

- Mean Life: The average time that the MRGB is expected to operate at a given stress level before failure. This metric is often referred to as mean time to failure (MTTF) or mean time before failure (MTBF).

- Failure Rate: The number of failures per unit time that can be expected to occur for the MRGB at a given stress level.

- Warranty Time: The estimated time when the reliability will be equal to a specified goal at a given stress level.

- $\mathbf{B}(\mathbf{X})$ Life: The estimated time when the probability of failure will reach a specified point $(\mathrm{X} \%)$ at a given stress level.

- Acceleration Factor: A unitless number that relates the MRGB's life at an accelerated stress level to the life at the use stress level.

- Probability Plot: A plot of the probability of failure over time. This can display either the probability at the use stress level or, for comparison purposes, the probability at each test stress level.

\section{Discussion}

This paper targets the MRGB for the maintenance department, to assist them into having a reliability prediction system that can predict probability of MRGB failures rather than unexpected ones. In the fight against MGRB failure and unreliability, a single CM technique should not stand alone. Numerous benefits are gained from integrating the oil analysis and the vibration analysis which are highly complementary. They reinforce indications seen in each technology, and have unique diagnostic strengths in highlighting specific fault conditions. The enhanced oil analysis provided further insight on the fluid properties, contamination and wear debris analysis, providing quick and reliable information on MRGB. Integration of these two CM techniques provided comprehensive insight into the true operating condition of the MRGB. Both oil analysis and vibration analysis can allow early detection and trending of numerous problems to which MRGB can be subjected. Detecting the faults is the first step in the diagnostic process. Early fault detection yields benefits in diagnostic time, avoidance of unplanned down-time, elimination of chain reaction failures, and improved precision of maintenance and operations decision making and actions. Eventually, confidence in maintenance and operations decisions can be substantially improved when the comprehensive CM and QALT method are employed. The probability of failure on MRGB and other helicopter critical components, in the future, is entrusted to make reliability a factor of paramount importance. This is due to the fact that the failure of any helicopter component will have more negative consequences. 


\section{Conclusion}

The combination of proactive and predictive maintenance strategies for the MRGB improves MAF control of failure root causes and its ability to diagnose MRGB failure conditions. This paper presents results from analyzing both the enhanced oil data from the laboratory, and the vibration data collected from the MGRB during actual maintenance flights. The probability of failure of the MRGB can now be anticipated by applying QALT analysis principles based on the computed time-to-failure information analyzed from both data. This study utilizes oil analysis and vibration analysis to obtain the reliability information much needed by the end user. The focus is making good maintenance decisions. The scarcity of information leads to decision uncertainty. Conversely, the presence of information improves the certainty in which decisions are made, thus improving the quality of decision making of the NURI maintenance department.

\section{References}

(1) Muhamad Murrad, Integration Of Vibration And Oil Analysis For Condition Monitoring Of Helicopter Main Rotor Gearboxes, Fifth DSTO International Conference on Health and Usage Monitoring, Melbourne, 2007

(2) ReliaSoft Corporation, Advanced Quantitative Accelerated Life Testing Analysis, RS 521 Seminar, Tucson, Arizona, 6-8 December 2006

(3) Noria Corporation, Oil Analysis Level $1 \& 2$, Certification Series Seminar, Kuala Lumpur, 18-20 September 2006

(4) Drew Troyer and Jim Fitch, Oil Analysis Basics, Noria Corporation, 2001

(5) ISVR, Advanced Structural Dynamics, Short Course, University of Southampton, 19-23 September 2005

\section{Acknowledgements}

We would like to thank the following people for their assistance in providing input to this paper:

(1) Tan Chek Zin (Institute of Noise and Vibration, UTM).

(2) Lt Col Samir RMAF (CAESE).

(3) Capt Azizul RMAF (10 ${ }^{\text {th }}$ Squadron NURI).

(4) Roslan, Yazid, CK Sam, Fhadilah, Hj Karim, Razali, Shahrizal and Hamid (STRIDE). 
Appendix

\section{Results of the Analysis}

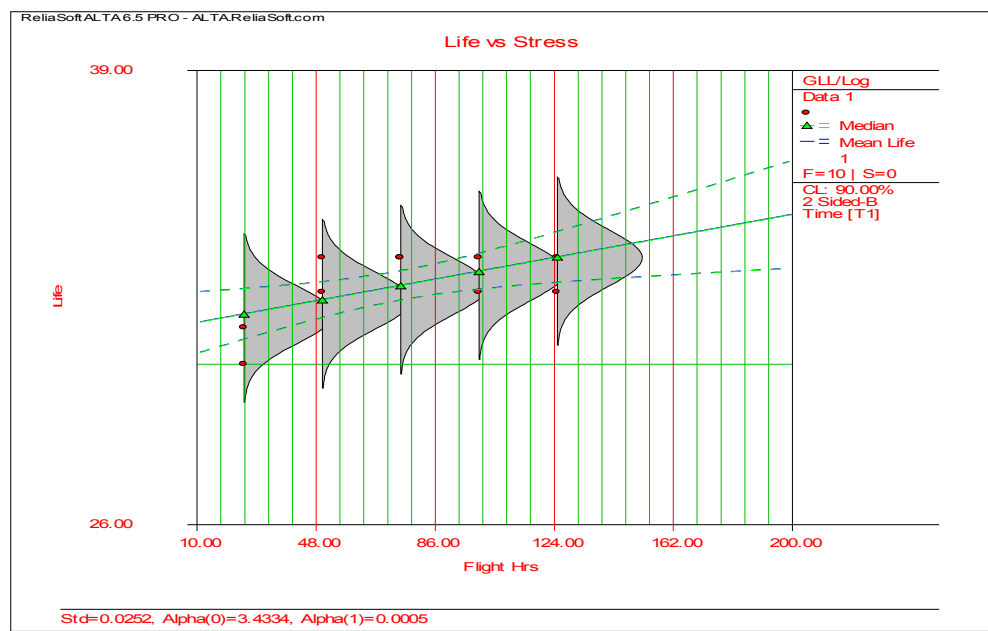

\section{User Input}

Flight Hrs $=2000$

\section{Parameter Values}

$\sigma_{T^{\prime}}=0.0252$

$\alpha_{j=1}=3.4334$

$\alpha_{j=2}=0.0005$

\section{Output}

Prob. of Failure $=\mathbf{0}$

Fig 2 Viscosity $40^{\circ} \mathrm{C} v s$. Flight Hrs

Brief Explanation for Figure 2 (the same for Figures 3-11) Once the parameters of the model ( $\sigma_{T}$, and $\alpha_{j}$ from Equation 3 ) are estimated, extrapolation and other life measures can be directly obtained using the appropriate equations. Using the Maximum Likelihood Estimation (MLE) method, confidence bounds for all estimates can be obtained. The more distant the accelerated stress from the operating stress, the greater the uncertainty of the extrapolation. The degree of uncertainty is reflected in the confidence bounds. The probability of failure computed for viscosity until 2000 flight hours is $0 \%$ although a slight increase in the characteristic is noted. This is only a probability and subsequent data after 125 flight hours may affect the percentage. No maintenance action may be required based on viscosity.

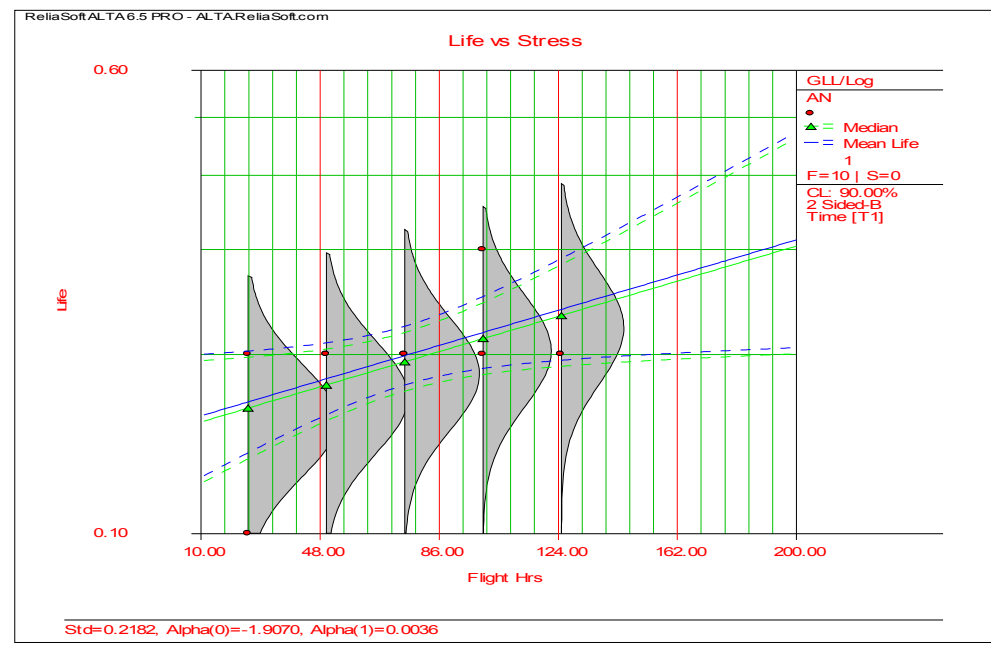

\section{User Input}

Flight Hrs $=2000$

\section{$\underline{\text { Parameter Values }}$}

$\sigma_{T^{\prime}}=0.2182$

$\alpha_{j=1}=-1.9070$

$\alpha_{j=2}=0.0036$

\section{Output}

Prob. of Failure $=\mathbf{0}$

Fig 3 Acid Number $v s$. Flight Hrs 


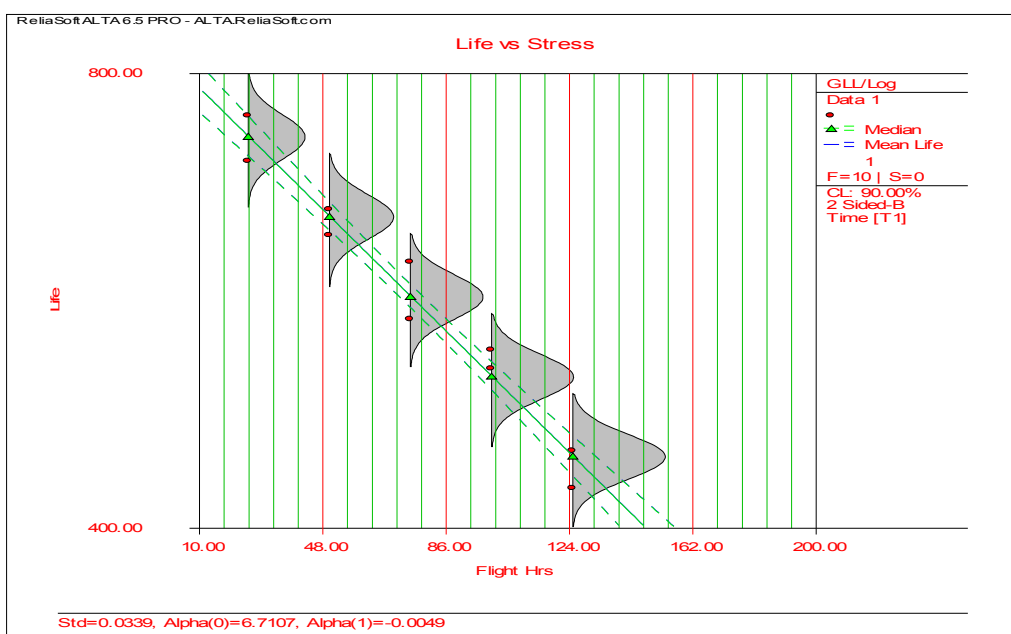

User Input

Flight Hrs $=2000$

$\underline{\text { Parameter Values }}$

$\sigma_{T^{\prime}}=0.0339$

$\alpha_{j=1}=6.7107$

$\alpha_{j=2}=-0.0049$

\section{Output}

Prob. of Failure $=\mathbf{0}$

Fig 4 Moisture vs. Flight Hrs

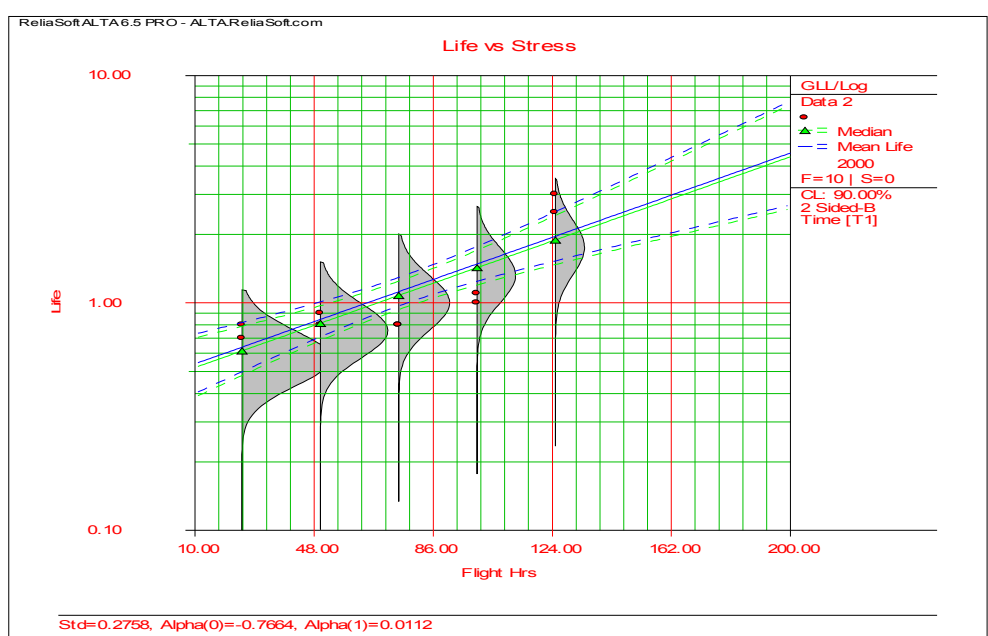

\section{User Input}

Flight Hrs $=2000$

Parameter Values

$\sigma_{T^{\prime}}=0.2758$

$\alpha_{j=1}=-0.7664$

$\alpha_{j=2}=0.0112$

\section{Output}

Prob. of Failure $=\mathbf{0 . 9 9 5 2}$

Fig 5 Wear Metal: Fe vs. Flight Hrs

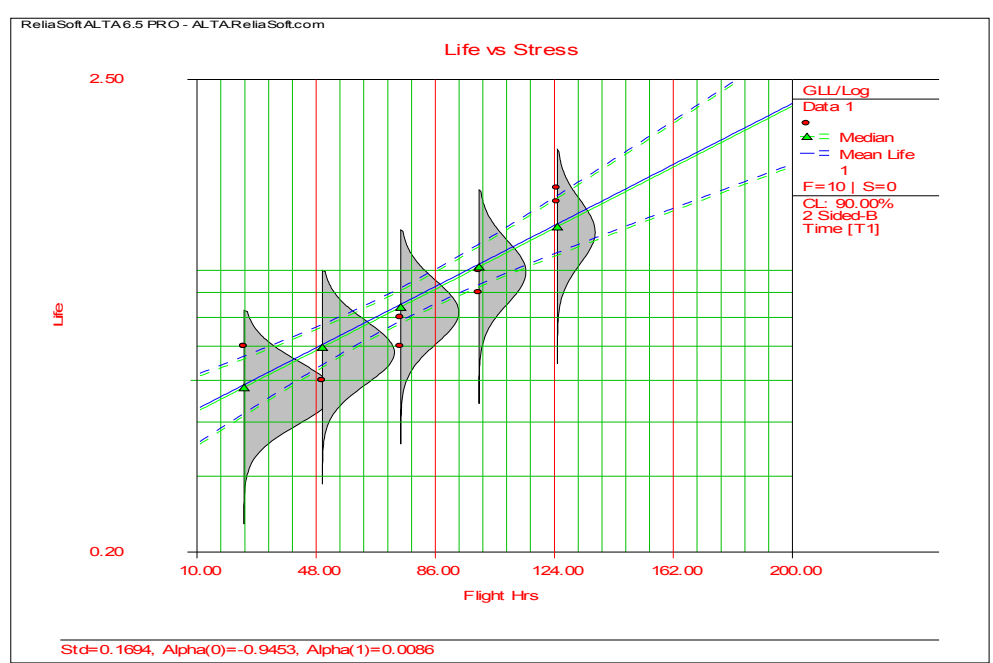

\section{User Input}

Flight Hrs $=2000$

$\underline{\text { Parameter Values }}$

$\sigma_{T^{\prime}}=0.1694$

$\alpha_{j=1}=-0.9453$

$\alpha_{j=2}=0.0086$

\section{Output}

Prob. of Failure $=\mathbf{0 . 9 9 9 9}$

Fig 6 Wear Metal: Sn vs. Flight Hrs 


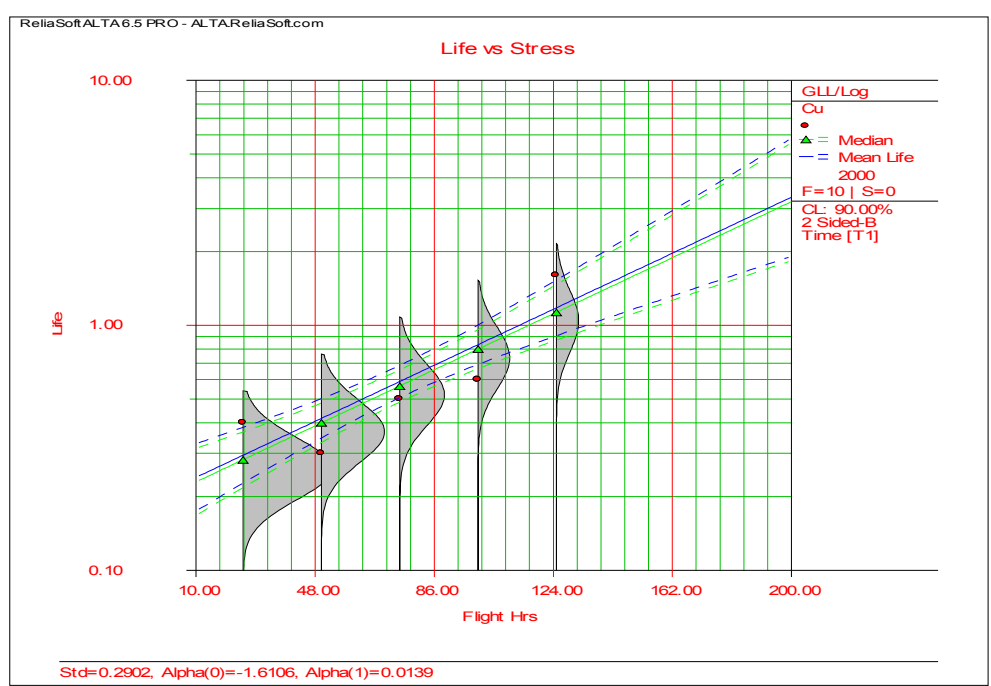

User Input

Flight Hrs $=2000$

Parameter Values

$\sigma_{T^{\prime}}=0.2902$

$\alpha_{j=1}=-1.6106$

$\alpha_{j=2}=0.0139$

\section{Output}

Prob. of Failure $=\mathbf{0 . 9 9 9 8}$

Fig 7 Wear Metal: Cu vs. Flight Hrs

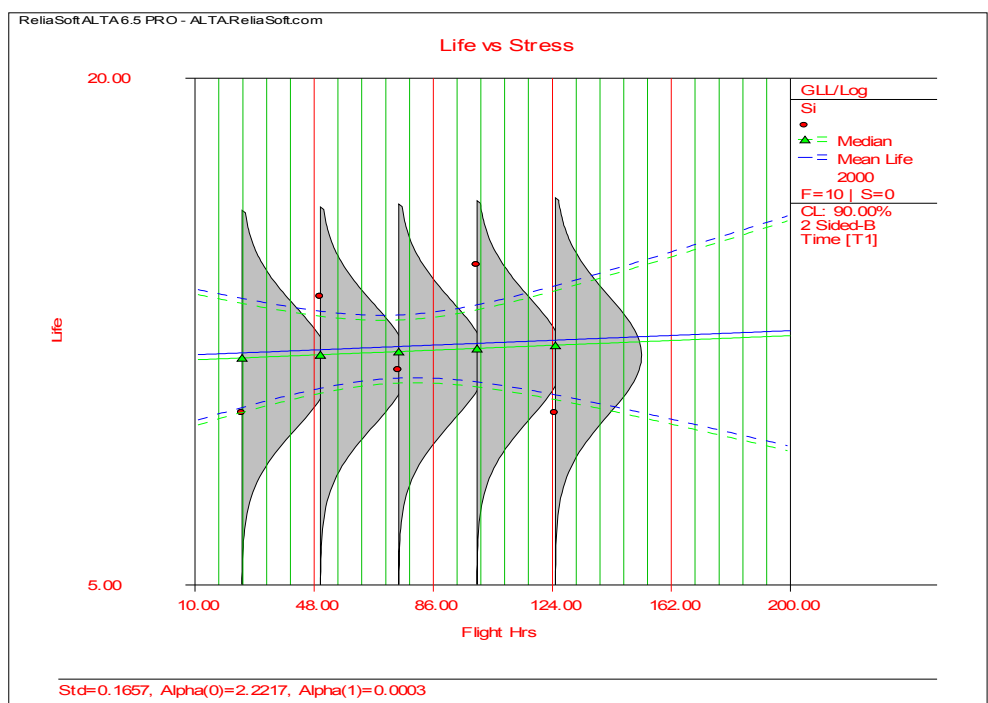

User Input

Flight Hrs $=2000$

Parameter Values

$\sigma_{T^{\prime}}=0.1657$

$\alpha_{j=1}=2.2217$

$\alpha_{j=2}=0.0003$

\section{Output}

Prob. of Failure $=\mathbf{0}$

Fig 8 Contaminant: Si $v s$. Flight Hrs

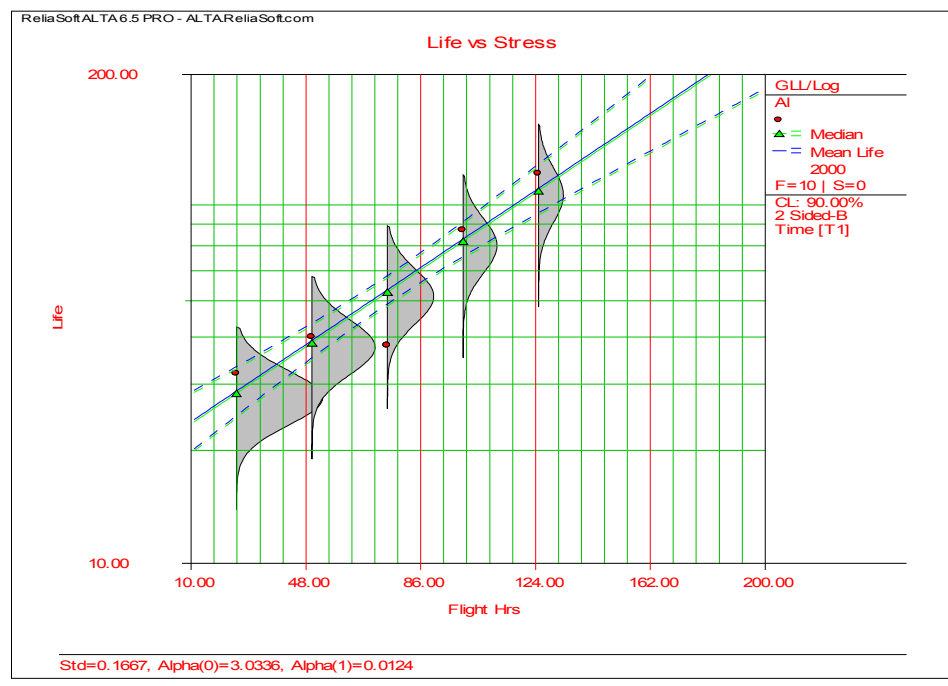

\section{User Input}

Flight Hrs $=2000$

$\underline{\text { Parameter Values }}$

$\sigma_{T^{\prime}}=0.1667$

$\alpha_{j=1}=\quad 3.0336$

$\alpha_{j=2}=0.0124$

\section{Output}

Prob. of Failure $=1.0000$

Fig 9 Contaminant: Al vs. Flight Hrs 


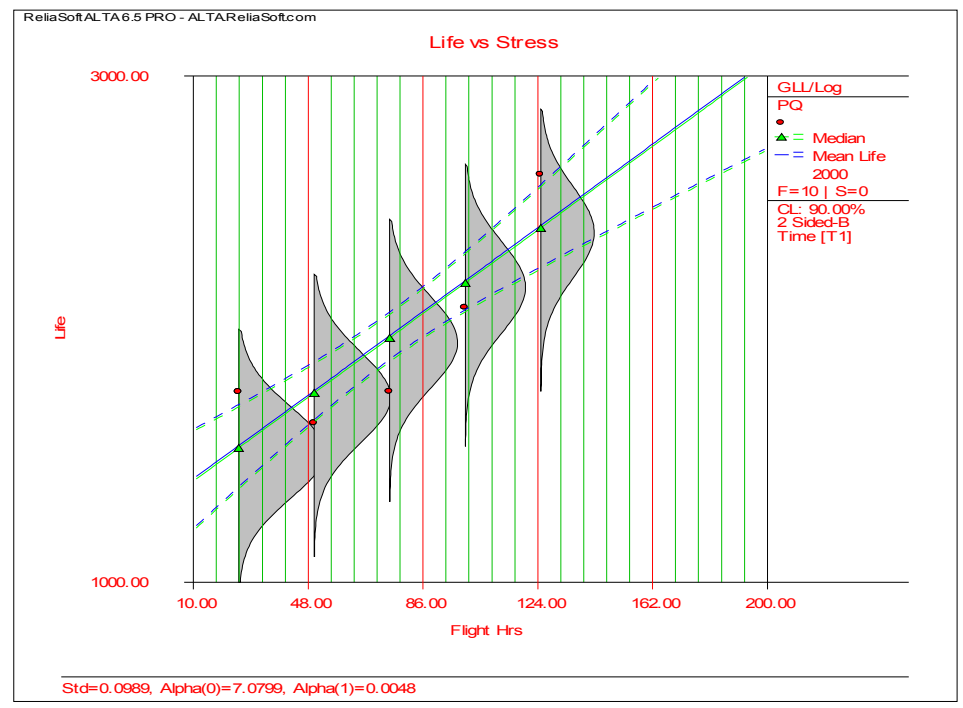

User Input

Flight Hrs $=2000$

$\underline{\text { Parameter Values }}$

$\sigma_{T^{\prime}}=0.0989$

$\alpha_{j=1}=7.0799$

$\alpha_{j=2}=0.0048$

\section{Output}

Prob. of Failure $=\mathbf{0 . 9 9 1 5}$

Fig 10 Particle Count vs. Flight Hrs

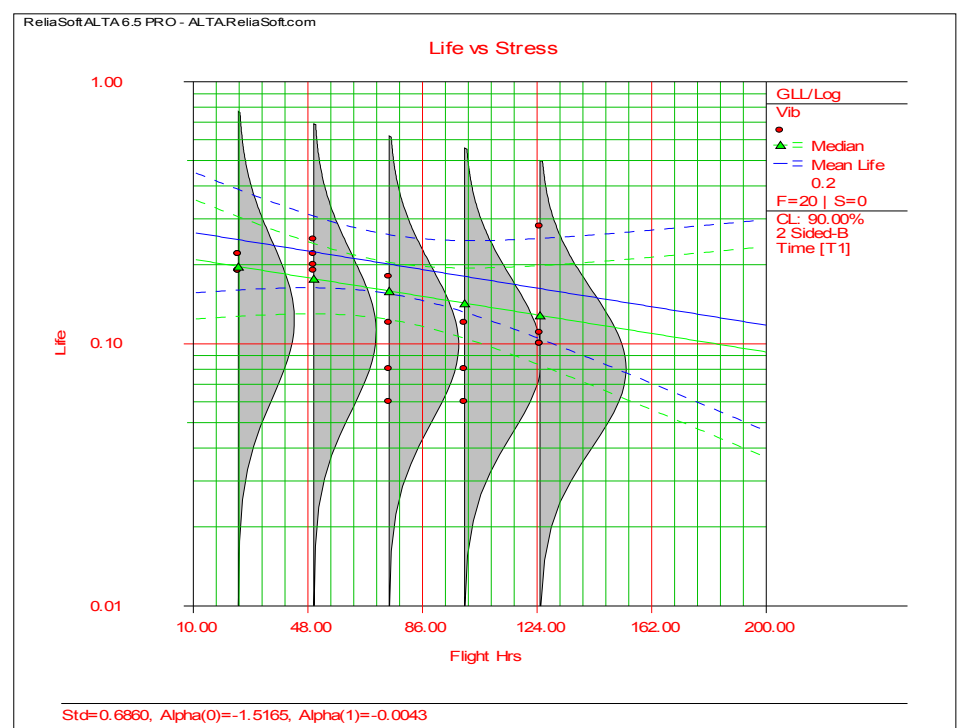

\section{User Input}

Flight Hrs $=2000$

$\underline{\text { Parameter Values }}$

$\sigma_{T^{\prime}}=0.6860$

$\alpha_{j=1}=-1.5165$

$\alpha_{j=2}=-0.0043$

\section{Output}

Prob. of Failure $=0.9867$

Fig 11 Vibe Measurement vs. Flight Hrs 